\title{
Equilibrio competitivo en Liga española de futbol de Primera División: Un test de Montecarlo basado en datos funcionales
}

\section{FRANCISCO MONTES}

Departamento de Estadística e I.O., UNIVERSIDAD DE VALENCIA, ESPAÑA. E-mail: francisco.montes@uv.es

\author{
RAMÓN SALA \\ Departamento de Matemáticas para la Economía y la Empresa, UNIVERSIDAD DE \\ VALENCIA, ESPAÑA. E-mail: ramon.sala@uv.es
}

\begin{abstract}
RESUMEN
La supremacía de unos pocos equipos sobre el resto de los participantes, es un factor común a las ligas europeas de futbol más conocidas. La española no es una excepción como demuestra el análisis que proponemos. Se analizan las clasificaciones de las últimas 10 temporadas, de la 2002-03 a la 2011-12, utilizando para ello técnicas del análisis de datos funcionales $(f d a)$. No solo el empleo de estas técnicas diferencia este trabajo de otros similares, otro rasgo distintivo lo constituye el uso de una distribución de probabilidad no uniforme sobre los tres posibles resultados de un partido, obtenida a partir de los resultados de los 3800 partidos correspondientes a las 10 temporadas examinadas y de la diferencia de categoría entre los equipos que se enfrentan. Un test de Montecarlo permite contrastar las hipótesis de uniformidad y no uniformidad en los resultados.
\end{abstract}

Palabras clave: Equilibrio competitivo, test de Montecarlo.

\section{Competitive Balance in the First Division Spanish Soccer League: A Montecarlo Test on Functional Data}

\begin{abstract}
The supremacy of a few teams over the other participants is a common factor in the major European football leagues. The Spanish First Division league is not an exception. In order to demonstrate this fact, functional data analysis is used to analyze football league classifications for last ten seasons, 2002-03 to 2011-12. Not only the use of these techniques distinguish this work from similar, another distinctive feature is the use of a non-uniform probability distribution on the three possible outcomes of a match, obtained from the results of the 3800 matches of the 10 seasons taking into account the difference between the categories of the teams in the match. A Monte Carlo test allows to test the hypotheses of uniformity and non-uniformity in the results.
\end{abstract}

Keywords: Competitive Balance, Montecarlo Test.

Clasificación JEL: C15, L83

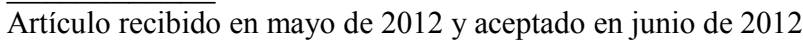

Artículo disponible en versión electrónica en la página www.revista-eea.net, ref. ə-30212 


\section{INTRODUCCIÓN}

El equilibrio entre los equipos que participan en una competición deportiva supone la existencia de igualdad de fuerzas entre ellos e implica una incertidumbre máxima en los resultados de sus enfrentamientos. Desde el punto de vista deportivo la incertidumbre de la clasificación final aumenta el interés de los aficionados. Los aspectos económicos que de ello se derivan no son de menor interés, tanto por la asistencia de espectadores a los estadios como por los previsibles ingresos de las televisiones.

La teoría del equilibrio competitivo entre los equipos fue desarrollada por Rottenberg (1956) y ha sido objeto de posteriores desarrollos teóricos (Scully, 1989, 1995; Dobson y Goddard, 2001) y de aplicaciones practicas. Podemos citar entre ellas las de Vrooman (1995) y Eckard (2001) en el campo del beisbol y las de Neale (1964) y Szymanski (2001) en el del futbol.

Distintos son los métodos empleados por los autores para contrastar el equilibrio competitivo. Dobson and Goddard (2004) emplean un modelo polinomial para establecer las tendencias en las competiciones inglesa y escocesa, detectando que el desequilibrio aparece entre los años 70 y 80 como consecuencia de la movilidad de los jugadores y los cambios en los contratos, que conducen a un desplazamiento de los buenos jugadores hacia los equipos con mayores recursos. Buzzacchi et al. (2003) obtienen la distribución de probabilidad asociada al numero de equipos distintos que han ocupado las $k$ primeras posiciones en una liga a lo largo de $T$ temporadas. La distribución se calcula bajo el supuesto de equilibrio perfecto entre los $n$ equipos participantes. Concluyen los autores que las ligas cerradas, sin promociones ni ascensos, son más equilibradas que las ligas abiertas con promociones y ascensos.

No existe, hasta donde llega nuestro conocimiento, un estudio similar para la liga española de primera división. Lo que no nos impide prever cual será el resultado: un patente desequilibrio. A pesar de este previsible resultado, el presente artículo se ocupa de ello por dos razones: la introducción de una nueva herramienta basada en el análisis de datos funcionales para llevar a cabo el estudio y un test de Montecarlo basado en la distancia entre funciones y el uso de una distribución no uniforme sobre las opciones de victoria por parte de los equipos a la hora de contrastar las hipótesis de equilibrio. Estrictamente hablando, y de acuerdo con este segundo aspecto, no se contrasta la hipótesis de equilibrio competitivo, sino la existencia de un desequilibrio mayor que el observado a lo largo de las 10 temporadas analizadas.

El artículo se estructura como sigue. La Sección 2 describe someramente las técnicas de análisis de datos funcionales $(f d a)$ que vamos a utilizar. La Sección 3 se ocupa de los datos objeto del estudio resumiendo la información que consideramos relevante. La Sección 4 muestra los resultados de aplicar las técnicas 
de $f d a$ a la liga española, la Sección 5 describe el test de Montecarlo y, por último, la Sección 6 presenta los conclusiones que de estos resultados se derivan.

\section{DETECCIÓN DE OUTLIERS EN DATOS FUNCIONALES}

La clasificación al final de una temporada cualquiera de la liga de futbol puede ser considerada como una función discreta. Si $x=1,2, \ldots, n$, donde $n$ es el número de equipos en competición, la función $y(x)$ representa la puntuación obtenida por el equipo que ha ocupado la $x$-ésima posición en la tabla. En un reciente trabajo, Hyndman y Sang (2010) presentan métodos gráficos para la representación de datos funcionales mediante la librería rainbow de R (Sang y Hyndman, 2010), que permiten la detección de outliers y que serán utilizados posteriormente.

\subsection{Diagrama funcional basado en la profundidad (funcional bagplot)}

El primero de ellos, denominado bivariate bagplot por los autores, está basado en la noción de bagplot introducido por Rousseeuw et al. (1999). Se parte de la noción de profundidad semiespacial (Tukey, 1975), que mide el grado de centralidad de un punto $\theta \in R^{2}$ con respecto a una nube puntos, $Z=\left\{z_{1}, z_{2}, \ldots, z_{n}\right\}$. La profundidad semiespacial de $\theta$ con respecto a $Z$, ldepth $(\theta, Z)$, se define como el menor número de puntos de $Z$ que caen en cualquier semiplano cerrado determinado por una recta que pasa por $\theta$, con lo que $0 \leq$ ldepth $(\theta, Z) \leq n$. La región de profundidad $k, D_{k}$, es el conjunto de todos los $\theta$ con ldepth $(\theta, Z) \geq k$. La regiones de profundidad son polígonos convexos que verifican $D_{k+1} \subset D_{k}$. A partir de estos elementos se obtiene el bagplot que consta de tres partes:

- la mediana, el punto $\theta$ con mayor $\operatorname{ldepth}(\theta, Z)$ o, en caso de no ser un único punto, el centro de gravedad de la región más profunda.

- el saco (bag), aquella región $B$ comprendida entre dos regiones consecutivas, $D_{k}$ y $D_{k-1}$ tales que $\# D_{k} \leq[n / 2] \leq \# D_{k-1}$, y que se obtiene por interpolación lineal tomando como referencia la mediana, y

- la corteza (fence), una región obtenida mediante una homotecia de factor $\rho$ del saco, tomando siempre como referencia la mediana.

Hyndman y Sang (2010) aplican la anterior construcción al conjunto $Z$ formado por los puntos cuyas coordenadas son los scores de las dos primeras componentes principales de la familia de funciones. En su trabajo original, Rousseeuw et al. (1999) utilizan $\rho=3$ pero Hyndman y Sang (2010) proponen 
tomar $\rho=2.58$ porque así, si los scores bivariantes siguen una distribución normal, la corteza contendrá el $99 \%$ de las observaciones.

Puesto que cada punto representa una función, las gráficas de las funciones contenidas en el saco y la corteza dan lugar a lo que los autores denominan functional bagplot, una región del plano constituida por la curva mediana, la correspondiente al punto más profundo, una región interior definida por las gráficas de las curvas correspondientes a los puntos del saco, y que son el 50\% del total, y una región exterior definida por las gráficas de las curvas correspondientes a los puntos de la corteza.

\subsection{Diagrama funcional basado en la densidad (funcional HDR boxplot)}

Este nuevo gráfico está basado en el diagrama bivariante de regiones de alta densidad (bivariate HDR boxplot) propuesto por Hyndman (1996), aplicado también a los scores de las dos primeras componentes principales de la familia de funciones. Estimada una densidad, $\hat{f}($.) a partir del conjunto de dichos scores, $Z$, la región de alta densidad (HDR) del $100(1-\alpha) \%$ se define como $R_{\alpha}=\left\{z ; \hat{f}(z) \geq p_{\alpha}\right\}$ donde $p_{\alpha}=\int_{R \alpha} \hat{f}(z) d z=1-\alpha$. Se trata, en definitiva, de la región con probabilidad de cubrimiento $1-\alpha$ en cuyos puntos la densidad estimada es mayor que en cualquiera de los puntos de su complementario. En el caso bivariante las HDR son contornos que crecen a medida que $\alpha$ disminuye.

En el bivariate HDR boxplot suelen representarse los conjuntos correspondientes a $\alpha=0.5$ (interna) y $\alpha=0.01$ (externa). El punto de mayor densidad, equivalente al más profundo en el bivariate bagplot, es ahora la moda, definida

como $\left\{z_{m} ; \hat{f}(z)=\sup _{z \in z} \hat{f}(z)\right\}$. Una segunda gráfica, el functional HDR boxplot, se obtiene al representar las funciones correspondientes a los puntos contenidos en sus dos conjuntos.

\subsection{Detección de outliers}

La detección de los outliers es una tarea importante para evitar los errores de de predicción (Catalán y Trívez, 2006). Como señalan Hyndman y Sang (2010), ambos tipos de gráficos permiten identificar las funciones outliers. Serán aquellas que quedan fuera de las regiones exteriores. En su artículo comparan los dos métodos anteriores con los propuestos por otros autores (Febrero et al., 2007; Hyndman y Ulla, 2007; Filzmoser et al., 2008) utilizando datos de mortalidad masculina en Francia en el periodo 1899-2005 y datos de la temperatura media mensual de la superficie del mar en la región de El Niño, en el periodo enero 1951 a diciembre de 2007. Concluyen los autores que, teniendo en cuenta el 
tiempo de computación y el número de outliers correctamente detectados, los dos métodos propuestos se comportan mejor que los restantes.

\section{DATOS}

Disponemos de una base de datos con los resultados de los 3800 partidos de la Liga española de futbol de Primera división correspondientes a las diez últimas temporadas, de la 2002-03 a la 2011-12. Para estudiar el equilibrio competitivo la información más relevante es la clasificación al final de cada temporada, que se recoge en la Tabla 1. En ella no aparecen los equipos porque no importa qué equipo ocupó qué posición sino el número de puntos conseguido en cada posición de la tabla.

Tabla 1

Clasificación final en las temporadas 2002/03 a 2011/12

\begin{tabular}{|c|c|c|c|c|c|c|c|c|c|c|}
\hline Clasificación & $\begin{array}{l}\text { ֻ̊․ } \\
\text { ஸे }\end{array}$ & $\begin{array}{l}\text { ષ્ڤ } \\
\text { ભ }\end{array}$ & $\begin{array}{l}n \\
0 \\
0 \\
0\end{array}$ & $\begin{array}{l}\circ \\
\text { 이 } \\
\text { மீ }\end{array}$ & $\begin{array}{l}\text { ó } \\
0 \\
0\end{array}$ & $\begin{array}{l}\infty \\
\stackrel{1}{1} \\
0\end{array}$ & $\begin{array}{l}\text { ̊̊ } \\
\text { ○े } \\
0 \\
0\end{array}$ & 웅 & $\stackrel{\check{c}}{\sigma}$ & $\frac{\mathfrak{T}}{\check{T}}$ \\
\hline 1 & 78 & 77 & 84 & 82 & 76 & 82 & 87 & 99 & 96 & 100 \\
\hline 2 & 76 & 72 & 80 & 70 & 76 & 74 & 78 & 96 & 92 & 91 \\
\hline 3 & 72 & 71 & 65 & 69 & 71 & 64 & 70 & 71 & 71 & 61 \\
\hline 4 & 61 & 70 & 62 & 68 & 66 & 64 & 67 & 63 & 62 & 58 \\
\hline 5 & 60 & 56 & 61 & 68 & 62 & 61 & 65 & 62 & 58 & 56 \\
\hline 6 & 56 & 55 & 60 & 64 & 60 & 57 & 62 & 58 & 58 & 55 \\
\hline 7 & 55 & 55 & 58 & 57 & 60 & 56 & 58 & 56 & 58 & 54 \\
\hline 8 & 54 & 54 & 51 & 55 & 54 & 52 & 55 & 54 & 49 & 52 \\
\hline 9 & 52 & 52 & 51 & 54 & 52 & 50 & 51 & 47 & 47 & 50 \\
\hline 10 & 50 & 51 & 51 & 52 & 50 & 49 & 47 & 47 & 47 & 49 \\
\hline 11 & 47 & 51 & 50 & 46 & 49 & 48 & 46 & 44 & 46 & 47 \\
\hline 12 & 47 & 48 & 50 & 45 & 49 & 48 & 46 & 43 & 46 & 47 \\
\hline 13 & 46 & 48 & 47 & 43 & 47 & 46 & 44 & 42 & 45 & 47 \\
\hline 14 & 46 & 47 & 47 & 42 & 46 & 46 & 43 & 41 & 45 & 46 \\
\hline 15 & 45 & 46 & 46 & 41 & 42 & 44 & 43 & 40 & 45 & 43 \\
\hline 16 & 44 & 43 & 44 & 40 & 40 & 43 & 43 & 39 & 44 & 43 \\
\hline 17 & 43 & 42 & 39 & 40 & 40 & 43 & 42 & 37 & 44 & 42 \\
\hline 18 & 36 & 41 & 37 & 39 & 39 & 42 & 42 & 36 & 43 & 41 \\
\hline 19 & 35 & 39 & 29 & 36 & 35 & 30 & 35 & 36 & 35 & 37 \\
\hline 20 & 32 & 26 & 28 & 24 & 28 & 26 & 33 & 34 & 30 & 27 \\
\hline
\end{tabular}

Fuente: Elaboración propia con datos de GECASport.

Como indicábamos anteriormente, cada clasificación puede ser considerada como una función discreta, $y(x)$, cuyo valor es el número de puntos conseguido por el equipo que finalizó la temporada en la posición $x$-ésima, $x=1,2, \ldots, 20$. Las gráficas de las 10 funciones se muestran en la Figura 1. 
Figura 1

Gráficos de las clasificaciones de las 10 emporadas

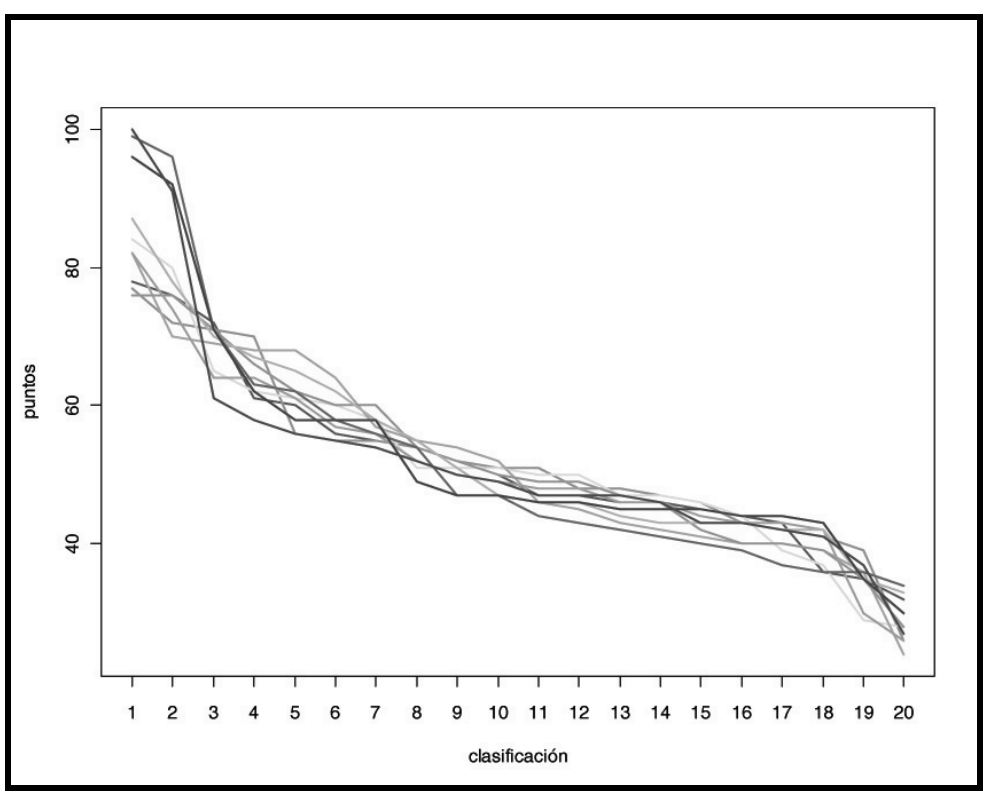

Fuente: Elaboración propia.

\section{RESULTADOS}

La aceptación o rechazo de la hipótesis de equilibrio competitivo exige comparar estas clasificaciones con las que obtendríamos bajo dicho supuesto, que implica una distribución uniforme sobre los tres resultados posibles de un partido: victoria local, victoria visitante o empate. Para ello simularemos 200 clasificaciones en estas condiciones y analizaremos las 210 funciones (200 clasificaciones simuladas +10 reales) con las herramientas de la sección anterior.

Como ya dijimos en la Introducción, el rechazo de la uniformidad es previsible, como así ocurre. Cualquier aficionado hubiera adelantado este resultado. Se hace necesario un análisis más realista que tome en consideración el desequilibrio entre los equipos y que ponga en evidencia, no la uniformidad, sino la existencia de temporadas (clasificaciones) que muestran un desequilibrio aún mayor que el observado. Esta nueva aproximación requiere en primer lugar medir el desequilibrio entre los equipos y, a continuación, estimar una distribución de probabilidad sobre los tres resultados de un partido que tenga en cuenta la categoría de los contendientes.

La Tabla 2 cumple con el primer requerimiento. Muestra todos los equipos que han participado en la Primera División en alguna de las 10 temporadas es- 
tudiadas. La segunda columna asigna a cada equipo una categoría de 1 (mayor) a 4 (menor). La asignación se ha llevado a cabo mediante un análisis cluster (véase, Pacheco y Valencia, 2005) en el que hemos hecho intervenir tres variables: el presupuesto, los puntos y los ingresos por derechos de TV, todas ellas acumuladas a lo largo de las 10 temporadas.

\section{Tabla 2}

Equipos que han jugado en Primera División a lo largo de las 10 temporadas y su categoría

\begin{tabular}{lc|lc}
\hline Equipo & Categoría & Equipo & Categoría \\
\hline Barcelona & 1 & Zaragoza & 3 \\
Real Madrid & 1 & Alavés & 4 \\
At. Madrid & 2 & Albacete & 4 \\
Ath. Bilbao & 2 & Almería & 4 \\
Valencia & 2 & Cádiz & 4 \\
Villarreal & 2 & Getafe & 4 \\
Betis & 3 & Gimnàstic Tarragona & 4 \\
Celta de Vigo & 3 & Granada & 4 \\
Deportivo & 3 & Hércules & 4 \\
Espanyol & 3 & Levante & 4 \\
Málaga & 3 & Murcia & 4 \\
Mallorca & 3 & Numancia & 4 \\
Osasuna & 3 & Rayo Vallecano & 4 \\
Racing de Santander & 3 & Recreativo de Huelva & 4 \\
Real Sociedad & 3 & Sporting de Gijón & 4 \\
Sevilla & 3 & Tenerife & 4 \\
Valladolid & 3 & Xerez & 4 \\
\hline
\end{tabular}

Fuente: Elaboración propia.

La distribución de probabilidad sobre los tres resultados posibles se muestra en la Tabla 3. Con el fin de hacer intervenir también la categoría de los equipos, estimamos una distribución de probabilidad condicionada a la diferencia de categoría entre el equipo local y el visitante (difcat en la tabla). Siendo cuatro las categorías, esta diferencia toma los 7 valores comprendidos entre -3 y 3 . Las marginales de la tabla contienen las frecuencias para los distintos resultados o diferencias de categorías. La estimación se ha llevado a cabo a partir de los resultados de los 3800 partidos correspondientes a las 10 temporadas analizadas, 2002-03 a 2011-12. 


\section{Tabla 3}

Probabilidades estimadas para los distintos resultados en función de la diferencia de categoría (difcat) ente los equipos contendientes

\begin{tabular}{c|ccc|c}
\hline difcat & visitante & empate & local & total \\
\hline-3 & 0,0610 & 0,0366 & 0,9024 & 82 \\
-2 & 0,1105 & 0,1630 & 0,7265 & 362 \\
-1 & 0,1929 & 0,2434 & 0,5637 & 871 \\
0 & 0,2658 & 0,2752 & 0,4590 & 1170 \\
1 & 0,3375 & 0,2583 & 0,4041 & 871 \\
2 & 0,4641 & 0,2928 & 0,2431 & 362 \\
3 & 0,6585 & 0,2317 & 0,1098 & 82 \\
\hline total & 1040 & 946 & 1814 & 3800 \\
\hline
\end{tabular}

Fuente: Elaboración propia.

\subsection{Simulaciones uniformes}

En este primer análisis partimos de una situación de perfecto equilibrio entre todos los equipos. Bajo este supuesto, hemos obtenido 200 clasificaciones correspondientes a otras tantas temporadas, simulando para ello los resultados de los 380 partidos de cada temporada. A las 200 funciones resultantes hemos añadido las clasificaciones de las 10 temporadas reales.

El resultado de cada partido se elige al azar entre los tres posibles, asignando a cada equipo la puntuación que se derive, 3 puntos al vencedor o 1 punto a cada equipo en caso de empate. Obsérvese que bajo el supuesto de uniformidad, no importan ni los equipos que contienden ni sus categorías. En consecuencia, las simulaciones son validas para cualquiera de las 10 temporadas, razón por la cual podemos analizarlas conjuntamente con las 200 simulaciones.

La Figura 2 muestra los diagramas bivariante HDR y bagplot correspondientes a las 210 funciones. De acuerdo con lo explicado en la Sección 2.2, el diagrama bivariante HDR (izquierda) muestra como outliers las temporadas 2008-09, 2009-10, el valor 20 corresponde a una de las clasificaciones simuladas y carece por tanto de interés para nosotros. Estas dos temporadas sobresalen del resto en las puntuaciones alcanzadas por los primeros clasificados (ver Tabla 1), que son superiores a las del resto de temporadas. Obsérvese también que la temporada 2009-10 muestra puntuaciones muy bajas entre las posiciones novena a decimoctava. Llama la atención, sin embargo, que habiéndose batido el record de puntos del primer clasificado en la última temporada, 2011-12, ésta no aparezca como outlier, es de hecho uno de los dos puntos en la pequeña bolsa gris al SO de la bolsa grande. Si utilizamos el método basado en la profundidad para detectar outliers, bagplot, vemos (Figura 2 derecha) que las temporada 2011-12 también es un outlier, así como la 2005-06 y la 2010-11. 
Figura 2

Diagrama bivariante HDR (izquierda) y diagrama bivariante bagplot (derecha) bajo el supuesto de uniformidad
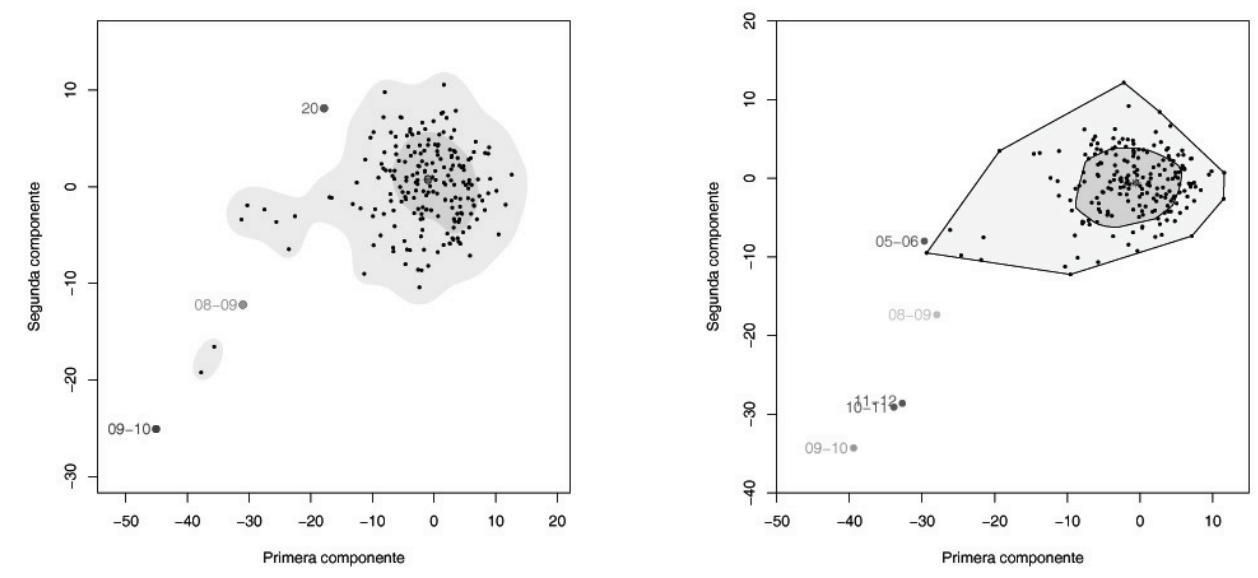

Fuente: Elaboración propia.

\subsection{Simulaciones ponderadas}

Este segundo análisis asume de entrada que el equilibrio competitivo no es real, haciendo depender el resultado del partido de la diferencia de categoría entre el equipo local y el visitante. Se utilizan ahora las probabilidades recogidas en la Tabla 3. Como consecuencia de este planteamiento las temporadas no son intercambiables, como ocurría anteriormente, porque que de una a otra se producen descensos y promociones y cambian los equipos y sus categorías. Cada temporada deberá ser analizada con las 200 simulaciones obtenidas a partir de las concretas diferencias de categorías de sus 380 partidos. En total serán ahora 201 funciones para cada temporada, las simuladas más la real de la temporada analizada.

Como era de esperar, los outliers han desaparecido pero no completamente. Las tres últimas temporadas, 2009-10, 2010-11 y 2011-12 continúan siendo outliers evidenciando un desequilibrio mayor del que supone la distribución de probabilidad de partida. La Figura 3 muestra los correspondientes gráficos. Aparecen en ella otros outliers correspondientes a clasificaciones simuladas que carecen de interés. 
Figura 3

Diagramas bivariante HDR bajo el supuesto de ponderación de las temporadas 2009/10, 2010/11 y 2011/12
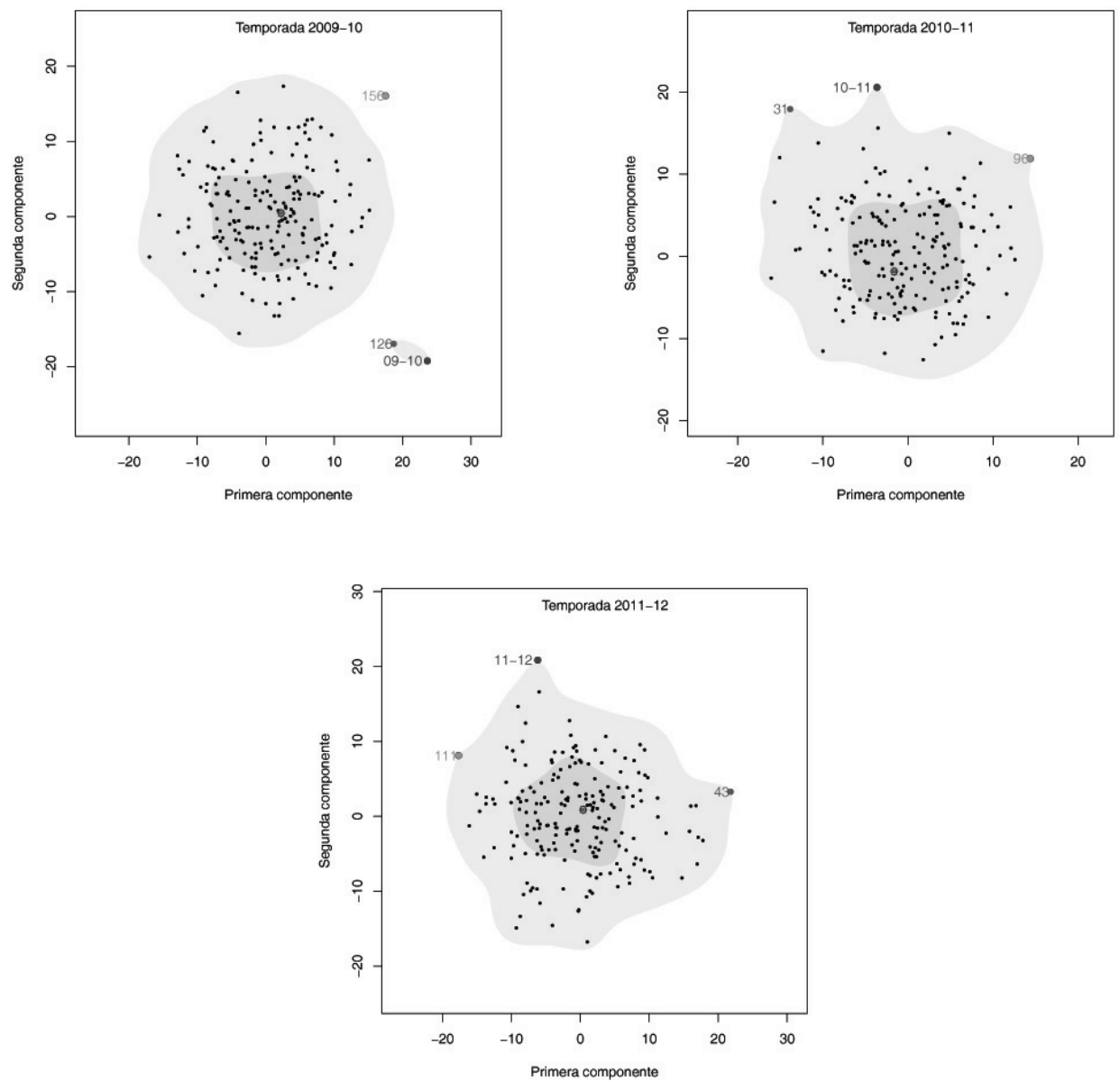

Fuente: Elaboración propia.

\section{UN TEST DE MONTECARLO}

La detección de outliers es un método indirecto para contrastar la hipótesis de probabilidad uniforme sobre el conjunto de resultados (equilibrio competitivo) o la de una distribución probabilidad bivariante sobre los resultados y la diferencia de categorías como la que expresa la Tabla 3. Es decir, el hecho de que las clasificaciones de algunas de las 10 temporadas analizadas sean outliers no nos permite en rigor rechazar las hipótesis nulas correspondientes. 
La construcción de un test apropiado choca con la dificultad de conocer la distribución de probabilidad del estadístico elegido, dificultad que puede soslayarse recurriendo a un test de Montecarlo. Lo primero será la elección del estadístico, para ello obtendremos la clasificación media, $y_{\text {media }}$, de las $n$ simulaciones obtenidas bajo un supuesto u otro. Como estadístico utilizaremos una distancia entre funciones,

$$
d_{i}=\left(\int_{D}\left(y_{\text {media }}(x)-y_{i}(x)\right)^{2} d x\right)^{\frac{1}{2}}
$$

Donde $D=[1,20]$ e $y_{i}$ es la clasificación obtenida para la temporada $i=1$, $2, \ldots, n+n_{r}$, con $n$ las simuladas y $n_{r}$ las reales. El valor de (1) se obtiene mediante aproximación numérica. Una vez ordenados los valores de $d_{i}$, si $c_{0}$ es posición de una de las 10 temporadas reales, su p-valor asociado vendrá dado por $\left(n+n_{r}-c_{0}\right) /\left(n+n_{r}\right)$.

Un test de Montecarlo similar, basado en la discrepancia entre funciones, es propuesto por Diggle (2003) para contrastar la uniformidad en un patrón de puntos.

\subsection{Hipotesis de equilibrio competitivo}

Se han eliminado al azar 10 de las 200 simulaciones, de forma que al añadir las 10 temporadas reales tenemos un total de $n+n_{r}=200$ valores de $d_{i}$. Las 10 temporadas presentan los 10 valores más grandes de $d_{i}$, tal como muestra la Tabla 4 en la que aparecen también sus posiciones y el p-valor asociado. La conclusión es por tanto que en las 10 temporadas no hay equilibrio competitivo puesto que la hipótesis nula de uniformidad ha de rechazarse.

\subsection{Hipotesis de desequilibrio}

A diferencia de la hipótesis de uniformidad y por la razones ya expuestas cuando se obtuvieron las simulaciones ponderadas, cada temporada requiere ahora su test específico. Para disponer de un total de 200 observaciones, eliminamos una al azar y añadimos la correspondiente a la temporada real $(n=199 \mathrm{y}$ $\left.n_{r}=1\right)$. La Tabla 4 recoge los resultados. Confirma el test lo que la detección de outliers permitía intuir, las tres últimas temporadas muestran un desequilibrio aún mayor que el que la distribución de probabilidad estimada en la Tabla 3 supone. No es el caso para el resto de temporadas todas con p-valores mayores que 0,05 . 


\section{Tabla 4}

Resultados del test de Montecarlo bajo las hipótesis de equilibrio y desequilibrio competitivo

\begin{tabular}{|c|ccc|ccc|}
\hline temporada & $\mathbf{d}_{\mathbf{i}}$ & $\begin{array}{c}\text { equilibrio } \\
\text { posición }\end{array}$ & p-valor & $\mathbf{d}_{\mathbf{i}}$ & $\begin{array}{c}\text { desequilibrio } \\
\text { posición }\end{array}$ & p-valor \\
\hline $2002-03$ & 22,8254 & 192 & 0,040 & 9,6806 & 59 & 0,705 \\
$2003-04$ & 23,6537 & 196 & 0,020 & 11,6588 & 137 & 0,315 \\
$2004-05$ & 23,8219 & 191 & 0,045 & 11,8061 & 108 & 0,460 \\
$2005-06$ & 26,1245 & 195 & 0,025 & 15,3844 & 182 & 0,090 \\
$2006-07$ & 28,7143 & 193 & 0,035 & 7,3195 & 5 & 0,975 \\
$2007-08$ & 29,8248 & 194 & 0,030 & 11,1758 & 95 & 0,525 \\
$2008-09$ & 31,1053 & 197 & 0,015 & 12,0212 & 120 & 0,400 \\
$2009-10$ & 39,3189 & 200 & 0,000 & 30,1100 & 200 & 0,000 \\
$2010-11$ & 41,3698 & 199 & 0,005 & 25,3149 & 200 & 0,000 \\
$2011-12$ & 47,9583 & 198 & 0,010 & 26,2858 & 199 & 0,005 \\
\hline
\end{tabular}

Fuente: Elaboración propia.

\section{CONCLUSIONES}

El objetivo de nuestro trabajo era comprobar que no existe equilibrio competitivo en la liga española de futbol de Primera División, al menos durante las 10 últimas temporadas que son las analizadas en este estudio. Para ello hemos considerado cada clasificación final como una función discreta definida sobre el conjunto $D=\{1,2, \ldots, 20\}$ y cuyos valores son los puntos alcanzados por cada uno de los 20 equipos. En una primera aproximación el desequilibrio se comprueba indirectamente mediante la detección de clasificaciones outliers. Un contraste más riguroso mediante un test de Montecarlo basado una distancia entre funciones (clasificaciones) confirma el desequilibrio. Ambas técnicas, detección de outliers y test de Montecarlo, se aplican a dos hipótesis nulas distintas: la primera se refiere al equilibrio en sentido estricto, todos los equipos son iguales y cualquier resultado de un partido es igualmente probable; la segunda admite ya de entrada un desequilibrio, el que supone asignar al resultado de cada partido una probabilidad en función de la diferencia de categoría de los equipos que compiten y que se recoge en la Tabla 3 . Los resultados del test se resumen en la Tabla 4 y rechazan el equilibrio en todas las temporadas, sin embargo, los resultados de las temporadas 2002-03 a 2008-09 son acordes con las probabilidades de la Tabla 3, no así las tres últimas temporadas, 2009-10 a 2011-12, que muestran un desequilibrio aún mayor del que aquellas probabilidades suponen.

No es casual que estas temporadas sean outliers y los tests confirmen no sólo el desequilibrio sino lo que podríamos denominar desequilibrio sobre el desequilibrio. Lo que destaca en todas ellas es la elevada puntuación alcanzada por los dos primeros equipos, a gran distancia de los restantes, especialmente en la temporada 2011-12 en la que se han batido dos records de puntos: un máximo de 100 para el primer clasificado y un mínimo de 61 para el tercero. No es ca- 
sualidad que los dos primeros clasificados sean el Real Madrid y FC Barcelona, equipos que han dominado la Liga española a lo largo de su historia. Existen además razones de índole económica para que el desequilibrio continúe y se agrande. Según datos del Deloitte Report (2012), ambos equipos encabezan la lista ingresos de todas las ligas europeas para la temporada 2010-11, con 479,5 millones de euros el Real Madrid y 450,7 millones de euros el FC Barcelona. Casi la mitad de estos ingresos los obtienen de los derechos de televisión. También en la distribución de este dinero el desequilibrio es bien patente, sólo ellos dos se reparten un $35 \%$ del total, otros dos equipos, At. Madrid y Valencia CF, se reparten un $11 \%$, distribuyéndose el $45 \%$ restante entre los otros 16 , lo que les supone apenas un 1,7\% de media. Es decir, entre aquellos y estos últimos la proporción es de 10 a 1. Esta desigualdad no se da en el resto de ligas europeas, en las que en el peor de los casos la ratio es de 2 a 1.

Una última consideración acerca del número de simulaciones, $n$, en los apartados 4.1, 4.2, 5.1 y 5.2. Los resultados se muestran robustos frente al valor de $n$. Con valores mayores, $n=500$, o menores, $n=100$, los únicos cambios que se observan son la aparición de distinto ruido, simulaciones que resultan ser outliers, y la inclusión como outliers de alguna de las 10 temporadas objeto de estudio; pero manteniéndose siempre las señaladas originalmente. Los test de Montecarlo no sufren variación significativa con uno u otro número de simulaciones.

\section{REFERENCIAS BIBLIOGRÁFICAS}

BUZZACCHI L., SZYMANSKI, S. Y VALLETTI, T. (2003) Equality of Opportunity and Equality of Outcome: Open Leagues, Closed Leagues and Competitive Balance. Journal of Industry, Competition and Trade, 3 (3), pp. 167-186.

DELOITTE (2012). Football Money League. http://www.deloitte.com/view/en_GB/ uk/industries/sportsbusinessgroup/sports/football/deloitte-football-moneyleague/9db981f2bd415310VgnVCM1000001a56f00aRCRD.htm

DIGGLE, P. J. (2003) Statistical Analysis od Spatial Points Patterns. $2^{\text {nd }}$ Edition. Arnold, London.

DOBSON, S. Y GODDARD, J. (2001) The Economics of football. Cambridge University Press, Cambrigde (UK).

DOBSON, S. Y GODDARD, J. (2004) Revenue divergence and competitive balance in a divisional sports league. Scottish Journal of Political Economy , 51 (3), pp. 359-376.

CATALÁN, B Y TRÍVEZ, F.J. (2006) Efectos de los outliers aditivos en la predicción de la varianza condicional en un modelo Arch. Estudios de Economía Aplicada , 24 (1), pp. 531-43. 
ECKARD, E. W. (2001) Equality of Opportunity and Equality of Outcome: Open Leagues, Closed Leagues and Competitive Balance. Economic Inquiry , 39 (3), pp. 430-443.

FEBRERO, M., GALEANO, P. Y GONZÁLEZ-MANTEIGA, W. (2007) A functional analysis of NOx levels: location and scale estimation and outlier detection. Computational Statistics, 22 (3), pp. 411-427.

FILZMOSER, P., MARONNA, R. Y WERNER, M. (2008) Outlier identification in high dimensions. Computational Statistics and Data Analysis, 52 (3), pp. 16941711.

HYNDMAN, R. J. (1996) Computing and graphing highest density regions. The American Statistician, 50 (2), pp. 120-126.

HYNDMAN, R. J. Y ULLAH, M. S. (2007) Robust forecasting of mortality and fertility rates: A functional data approach. Computational Statistics and Data Analysis, 51 (10), pp. 4942-4956.

HYNDMAN, R. J. Y SHANG, H. L. (2010) Rainbow Plots, Bagplots, and Boxplots for Functional Data. Journal of Computational and Graphical Statistics, 19 (1), pp. 29-45.

NEALE, W. (1964) The peculiar economics of professional sports. Quarterly Journal of Economics, 78, pp. 1-14.

PACHECO, J. Y VALENCIA, G. (2005) Análisis de nuevos métodos de clasificación. Un ejemplo ilustrativo de su uso en la agrupación de los municipios de Castilla y León. Estudios de Economía Aplicada , 23 (3), pp. 711729.

ROTTENBERG, S. (1956) The baseball players labor market. Journal of Political Economy, 64(3), pp. 242-258.

ROUSSEEUW, P. J., IDA RUTS, I. Y TUKEY. J. W. (1999) The Bagplot: A Bivariate Boxplot. The American Statistician, 53(4), pp. 382-387.

SHANG, H. L. Y HYNDMAN, R. J. (2010) rainbow: Rainbow plots, bagplots and boxplots for functional data. R package version 2.3.1. http://CRAN.R-project.org/package=rainbow

SCULLY, G. W. (1989) The Business of Major League Baseball. The Chicago University Press, Chicago.

SCULLY, G. W. (1995) The Market Structure of Sports. The Chicago University Press, Chicago.

SZYMANSKI, S. (2001) Income inequality, competitive imbalance and the attractiveness of team sports: some evidences and a natural experiment from English soccer. Economic Journal, 111, F59-F84.

TUKEY. J. W. (1975) Mathematics and the picturing of data. In Proceedings of the International Congress of Mathematicians, Vancouver, pp. 523-531.

VROOMAN, J. (1995) A general theory of professional sports league. Southern Economic Journal, 61, pp. 971-990. 